\title{
Referentenentwurf zum PsychVVG: Wir sind auf einem guten Weg - Pro
}

\author{
Ministerial Draft Bill for the Law on Remuneration of Inpatient Psychiatry and \\ Psychosomatics (Psych VVG): We are Headed in the Right Direction - Pro
}

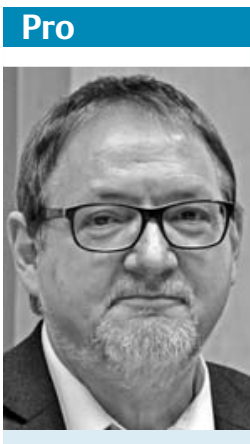

Dieter Grupp

Mit dem vom Bundesministerium für Gesundheit am 19.5. 2016 vorgelegten Referentenentwurf für ein Gesetz zur Weiterentwicklung der Versorgung und der Vergütung für psychiatrische und psychosomatische Leistungen (PsychVVG) wird eine grundlegende Richtungsänderung der zukünftigen Finanzierung von psychiatrischen und psychosomatischen Einrichtungen auf den Weg gebracht. Wie im Eckpunktepapier am 18.2.2016 bereits angekündigt hat das BMG damit die Kritik der Fachverbände, die im Rahmen eines strukturierten Dialoges im BMG aufgearbeitet wurden, aufgenommen und ein neues Entgeltsystem entworfen. Der Referentenentwurf stoppt die seit 1.1.2013 in der Bundespflegesatzverordnung vorgesehene Einführung eines Preissystems (PEPP) nach DRG-Logik, das nach gültigem Gesetz ab 1.1.2017 für alle psychiatrischen Krankenhäuser verbindlich geworden wäre.

\section{Weiterentwicklung der Versorgung \\ $\nabla$}

Der Gesetzesentwurf beschäftigt sich nicht nur in der Gesetzesüberschrift mit der Weiterentwicklung des psychiatrischen Versorgungssystems. Das vorgeschlagene Gesetz ist kein Kostendämpfungsgesetz und beschäftigt sich nicht ausschließlich mit der Finanzierung psychiatrischer und psychosomatischer Leistungen, sondern versucht ernsthaft an den Strukturen der sektorisierten Versorgung in Deutschland zu rütteln. Dazu wird der §39 SGB V „Krankenhausbehandlung“, der bisher die Möglichkeiten von Krankenhäusern einschränkte, außerhalb ihres stationären Versorgungsauftrags sektorübergreifend zu agieren, ergänzt durch eine „stationsäquivalente“ Behandlungsmöglichkeit. „Mobile ärztlich geleitete multiprofessionelle Behandlungsteams“ dürfen zukünftig außerhalb des Krankenhauses tätig werden und Patienten im direkten häuslichen Lebensumfeld behandeln. Das psychiatrische Krankenhaus darf zukünftig Leistungen erbringen, die bisher formal dem niedergelassenen Bereich vorbehalten waren. Die notwendige Dreiseitigkeit der Vereinbarungen (Krankenkassen, Krankenhausgesellschaft und Kassenärztliche Vereinigungen) wird dann im $§ 115$ d, der die Modalitäten einer derartigen mobil aufsuchenden Behandlung regelt, so formuliert, dass eine zu erwartende Blockadehaltung der Kassenärztlichen Bundesvereinigung von vornherein verhindert wird. Bei Nichteinigung wird die Ausgestaltung einer Schiedsstelle übertragen.

Diese scheinbar kleinen Veränderungen durchbrechen das starre deutsche, sektorisierte Gesundheitssystem (ähnlich wie die Einführung des ambulanten Operierens) und sind ein mutiger Schritt in Richtung sektorübergreifender Behandlung in der psychiatrischen Versorgung.

Es bleiben sicherlich bei der Ausgestaltung des sektorübergreifenden Ansatzes noch viele Wünsche offen. Im mit Lobbyisten besetzten Minenfeld zwischen Krankenkassen, Krankenhausgesellschaft und Kassenärztlicher Bundesvereinigung schafft das BMG aber Spielräume, die für echte Fortschritte in der psychiatrischen und psychosomatischen Versorgung sorgen können.

\section{Krankenhausindividuelle Budgets $\nabla$}

Der neue Gesetzesentwurf stellt krankenhausindividuelle Budgets in den Mittelpunkt. Das bedeutet eine deutliche Abkehr vom bisherigen DRG/PEPP-System und von der bisherigen Linie der Gesundheitspolitik. Die Grundidee, durch zentrale Steuerung und immer mehr Daten die
Versorgung der Patienten vor Ort zu verbessern, wird zumindest für die Psychiatrie infrage gestellt. Krankenhausindividuelle Budgets werden von den regionalen Verhandlungspartnern unter Berücksichtigung struktureller regionaler Besonderheiten festgelegt. Die Pluralität in der Versorgungslandschaft wird akzeptiert.

Die krankenhausindividuellen Budgets sind zunächst nicht gedeckelt! Vergleichswerte aus dem Krankenhausvergleich können regional überschritten werden, wenn die Gründe für die Überschreitung vom Krankenhaus schlüssig dargelegt werden.

Diese Formulierungen bieten viel Spielraum und damit natürlich auch ein großes Konfliktpotenzial für die Verhandlungen vor Ort. Dabei kommt dem vorgesehenen leistungsbezogenen Krankenhausvergleich eine entscheidende Rolle zu. Sind die Vergleichswerte „Grundlage“ oder „Orientierung“ für die regionalen Budgets? Sind Überschreitungen „unabweisbar“ oder nur „notwendig“? Die Vorgaben werden mit Sicherheit von den Kostenträgern anders interpretiert werden wie von den Leistungserbringern. Dieses Konfliktpotenzial bietet aber nicht nur Risiken für die Weiterentwicklung der Versorgung, sondern eben auch Chancen: Alles wird möglich.

Es liegt nun an den Fachgesellschaften und Leistungserbringern die Themen „strukturelle Besonderheiten“ und „Unabweisbarkeit von Budgetüberschreitungen“ entsprechend auszuformulieren.

\section{Verbindliche Mindestvorgaben für Personal \\ $\nabla$}

Der spannendste Teil der Budgetfindung dürfte das Thema „personelle Ausstattung“ sein. Als Nachfolgeregelung für die Personalverordnung Psychiatrie soll der Gemeinsame Bundesausschuss (GBA) verbindliche Mindeststandards für die Personalausstattung von psychiatrischen Einrichtungen festlegen. Diese Mindeststan- 
dards sind ab 2020 bei der Budgetfindung verbindlich zu berücksichtigen. Damit wird das normative Element der Personalverordnung Psychiatrie (Psych-PV) über verbindliche Qualitätsvorgaben quasi durch die Hintertür wieder eingeführt. Empirische IST-Personalbesetzungen bestehender Krankenhäuser sind somit nicht Basis des Budgets, sondern über Mindeststandards normativ vorgegebene Soll-Werte für eine Personalausstattung die zu einer leitliniengerechten Behandlung beitragen soll. Diese Regelung verdeutlicht die prinzipielle Abkehr des Referentenentwurfs von der bisherigen Linie der „Durchschnittspreisbildung“ mit der impliziten Spirale nach unten und den Willen, Möglichkeiten zu eröffnen, die bestehende psychiatrische Versorgung weiterzuentwickeln und zu verbessern.

Selbstverständlich lässt die Regelung viele Fragen offen. Was sind verbindliche Mindeststandards? Werden Durchschnittspersonalkosten finanziert oder reale ISTKosten vor Ort? Werden Tarifveränderun- gen adäquat berücksichtigt? Werden regionale „Rekrutierungsaufschläge“ auf die Tarifvergütung berücksichtigt? usw. Auch hier gilt, dass der Referentenentwurf Spielräume in der Budgetfindung eröffnet, die in den Verhandlungen vor Ort Chancen aber auch Risiken bergen.

Als Zugeständnis an die Kassenseite bleibt es beim PEPP-System als Abrechnungseinheit. Es werden Relativgewichte kalkuliert. Es werden Landes- und Bundesbasisentgeltwerte kalkuliert, die Einfluss auf die Budgetfindung nehmen werden. Ihnen fehlt jedoch die aus dem DRG und alten PEPP-System bekannte Verbindlichkeit für die Budgetfindung. Die Verantwortung der Verhandlungspartner vor Ort wird im Vergleich zu diesen Systemen erheblich gestärkt. Es wird darauf ankommen, dass die Krankenhäuser in diesen Verhandlungen ihre Spielräume nutzen und die Möglichkeiten zur Weiterentwicklung des psychiatrischen Versorgungssystems, die ihnen der Gesetzgeber jetzt eröffnet auch ergreifen.
Der vorliegende Referentenentwurf ist ein mutiger Schritt in die richtige Richtung. Er bietet viel Interpretationsspielraum und damit auch Möglichkeiten positive Entwicklungen anzustoßen.

Sie haben eine eigene Meinung zu diesem

Thema? Dann schreiben Sie uns an:

psychiat-praxis@thieme.de!

\section{Korrespondenzadresse}

\section{Dr. med. Dipl.-Psych. Dieter Grupp}

Geschäftsführer des Zentrums für Psychiatrie Südwürttemberg

Pfarrer-Leube-Straße 29

88427 Bad Schussenried dieter.grupp@zfp-zentrum.de

Bibliografie

DoI http://dx.doi.org/

10.1055/s-0042-112211

Psychiat Prax 2016; 43: 302-303

(C) Georg Thieme Verlag KG

Stuttgart · New York

ISSN 0303-4259 